\title{
ASYMPTOTICS OF A NONLINEAR RELATIVISTIC WAVE EQUATION ${ }^{1}$
}

\author{
BY C. S. MORAWETZ AND W. A. STRAUSS \\ Communicated by P. D. Lax, February 9, 1971
}

K. Jörgens [1] has proved the global existence of classical solutions of the Cauchy problem for

$$
u_{t t}-\Delta u+m^{2} u+u^{3}=0
$$

with $m \geqq 0$, in all space-time. I. Segal in [3] has proved the existence of the free-to-perturbed wave operators and in [4] the existence of the scattering operator on numerically small solutions. He has conjectured that the scattering operator exists in general. Segal's conjecture has been verified when $m=0$ in [5]. We have succeeded in proving the conjecture when $m>0$.

Definitions. By a free solution we mean a solution of the associated linear equation (equation $\left(^{*}\right.$ ) without $u^{3}$ ). The norm

$$
\|u\|^{2}=\sup _{t} \int\left(u_{t}^{2}+|\nabla u|^{2}+m^{2} u^{2}\right) d x+\sup _{x, t} u^{2}+\int_{\infty}^{\infty} \sup _{x} u^{2} d t
$$

is finite for all free solutions with smooth Cauchy data of compact support. Define $F$ to be the space of all their limits under this norm.

THEOREM 1. Any solution of $(*)$ with smooth Cauchy data of compact support tends to zero uniformly as $|t| \rightarrow \infty$. Furthermore, the solution approaches a free solution $u_{+}$in the energy norm as $t \rightarrow+\infty$ and a free solution $u_{-}$as $t \rightarrow-\infty$.

There are extensions of this theorem to: weak solutions, more general nonlinear terms, and a rate of decay as $|t| \rightarrow \infty$.

THEOREM 2. Whenever $u$ is a solution of $\left(^{*}\right)$ which tends to $u_{ \pm}$as above, we define the operator $S$ by $S\left(u_{-}\right)=u_{+}$. Then $S$ is defined on all of $F$ and is a homeomorphism of this space onto itself which preserves the energy norm.

The proofs are based on an estimate derived from [2], on some new

AMS 1970 subject classifications. Primary 35L30, 35L60, 35P25, $47 \mathrm{H} 99$.

1 This paper was supported in part by Contract No. DA-31-124-ARO-D-365 at New York University and NSF Contract No. GP 16919 at Brown University. 
estimates of the Riemann function of the linear equation, and on Segal's approach to the scattering problem.

\section{REFERENCES}

1. K. Jörgens, Das Anfangswertproblem im Grossen für eine Klasse nichtlinearer Wellengleichungen, Math. Z. 77 (1961), 295-308. MR 24 \#A323.

2. C. S. Morawetz, Time decay for the nonlinear Klein-Gordon equation, Proc. Roy. Soc. Ser. A 306 (1968), 291-296. MR 38 \#2455.

3. I. Segal, Quantization and dispersion for nonlinear relativistic equations, Proc. Conf. Mathematical Theory of Elementary Particles (Dedham, Mass., 1965), M.I.T. Press, Cambridge, Mass., 1966, pp. 79-108. MR 36 \#542.

4. - Dispersion for non-linear relativistic equations. II, Ann. Sci. École Norm. Sup. (4) 1 (1968), 459-497. MR 39 \#5109.

5. W. A. Strauss, Decay and asymptotics for $\square u=F(u)$, J. Functional Analysis 2 (1968), 409-457. MR $38 \# 1385$.

Courant Institute of Mathematical Sciences, New York University, New YoRK, NEW YORK 10012

Brown University, Providence, Rhode Island 02912 